\title{
Ai Weiwei: La recepción de su producción artística
}

\section{Ai Weiwei: The reception of his artistic production}

\author{
Laia Manonelles \\ Universidad de Barcelona. Universitat Oberta de Catalunya \\ laiam23@hotmail.com
}

Recibido: 30 de junio de 2014

Aprobado: 2 de agosto de 2014

\section{Resumen}

Actualmente Ai Weiwei es el creador con mayor proyección dentro y fuera de China, destacando especialmente por su prolífica, heterogénea, comprometida y polémica producción artística. Sus propuestas suscitan un gran interés y obtienen una destacada repercusión tanto en China como en un escenario internacional. Precisamente, es uno de los artistas contemporáneos chinos que ha logrado una mayor presencia en los medios de comunicación y en los espacios expositivos españoles, siendo un ejemplo de ello la organización de la primera exposición museística de su obra en el Centro Andaluz de Arte Contemporáneo (2013) y el estreno de la película documental sobre su vida y su obra Never Sorry (2013), dirigida por Alison Klayman.

Ai Weiwei es un autor mediático y su visibilidad deviene una plataforma desde donde articular proyectos artísticos que enfocan determinadas problemáticas sociales. A la vez, la crítica política de Ai Weiwei resulta especialmente atractiva en un contexto euroamericano que subraya su papel de disidente y agitador. Dentro de tales parámetros se ahondará en la recepción de la producción artística de Ai Weiwei en el marco internacional y en el contexto español, analizando la aproximación que la prensa esboza sobre dicho autor.

Palabras clave: Ai Weiwei, recepción artística, arte y política, activismo.

Manonelles, L. (2015): Ai Weiwei: La recepción de su producción artística. Arte, Individuo y Sociedad, $27(3) 413-428$

\begin{abstract}
Ai Weiwei is the artist with greater visibility inside and outside China, he stands out for his prolific, diverse, committed and controversial artistic production. His proposals raise considerable interest and obtain an outstanding impact in China and in an international scenario. Indeed, he is one of the Chinese contemporary artists that have achieved greater presence in the media and in the Spanish exhibition circuits, an example of this is the organization of the first museum exhibition of his work at the Centro Andaluz de Arte Contemporáneo (2013) and the premiere of the documentary film about his life and his work Never Sorry (2013), directed by Alison Klayman.

Ai Weiwei is a public figure and his visibility becomes a platform from which to articulate artistic projects that focus on specific social problems. At the same time, the political criticism of Ai Weiwei is especially attractive in a Euro-American context which emphasizes his role as a dissident and agitator. Within these parameters, we will delve into the reception of the artistic production of Ai Weiwei both in the international and the Spanish context, by analyzing the approach of the media about this author. Keywords: Ai Weiwei, artistic reception, art and politics, activism.
\end{abstract}


Sumario: 1. Introducción, 2. Arte y compromiso: proyectos colaborativos, 3. La recepción de la producción artística de Ai Weiwei, 3.1. La recepción de Ai Weiwei en el marco internacional y en el contexto español, 3.2. Resistencia y tradición en el CAAC de Sevilla, 4. Estrategias subversivas. 5. Conclusiones. Referencias.

Este artículo recoge resultados de la investigación de los proyectos de investigación GREGA/1 (HAR201124212/2012-2014, Universidad de Barcelona) e Interasia (Universidad Autónoma de Barcelona, FFI2011-29090 y 2014 SGR 1402), financiados por el Ministerio de Ciencia e Innovación

\section{Introducción}

Ai Weiwei es uno de los artistas contemporáneos que ha vivido la transición de la emergencia del arte vanguardista en China, a finales de la década de los setenta del pasado siglo, a la plena consolidación del arte experimental chino en el mercado y en el circuito expositivo internacional. Asimismo, actualmente, es el creador con mayor proyección dentro y fuera de China, destacando especialmente por su prolífica, heterogénea, comprometida y polémica producción artística.

Si esbozamos brevemente su biografía hay que apuntar que, después del período de represión maoísta, se matriculó en la Academia de Cine de Pekín, en 1978. Un año más tarde fue partícipe de la fundación del grupo de Las Estrellas (Xing Xing), un colectivo que empezó a experimentar con nuevos lenguajes vanguardistas como la Abstracción, el Surrealismo y el Dadaísmo. Con el movimiento de Las Estrellas comenzó a desmitificar los símbolos de poder comunista que le habían oprimido a él y a su familia, siendo un punto de inflexión en su trayectoria artística. Aquí es preciso mencionar que su padre fue el reconocido poeta Ai Qing (1910-1996), quien durante la Revolución Cultural fue acusado de reaccionario y enviado a un campamento de reeducación a Xinjiang junto a su familia. Ai Qing padeció maltratos además de realizar arduos trabajos, siendo Ai Weiwei testigo de tales vejaciones. Otro momento determinante en la gestación del trabajo conceptual de Ai Weiwei fueron los doce años que vivió en Nueva York, puesto que en 1981 emigró a los Estados Unidos y estudió en el centro Parson School of Design, profundizando en el Dadaísmo, el Arte Conceptual, el Minimalismo y el Pop art a la vez que entró en contacto con el poeta Allen Ginsberg, el pintor Keith Haring y el fotógrafo Robert Frank. El artista expone:

En Estados Unidos, aprendi sobre el arte conceptual, el minimalismo, el arte pop, y Marcel Duchamp, que me influyó profundamente. Aprendí a ser un artista inteligente, no un artista únicamente con habilidades visuales o técnicas. Éstas hacen falta, pero sólo como herramienta para representar tu idea (Reinoso, J., Ai Weiwei, 2009, 16 de mayo).

En relación a la influencia que han ejercido sobre él su progenitor y la estancia en los Estados Unidos comenta:

Estoy muy influenciado por el espiritu de mi padre, de hablar a favor de la verdad y aguantar las consecuencias, y siempre estar del lado de la gente, no del poder, no del gobierno. Esa ha sido su influencia. Pero por supuesto nunca nos habría animado... a involucrarnos en la política porque toda su vida estuvo perjudicada por eso.

Me daba miedo cuando era joven... cuando no tenía suficientes conocimientos, 
suficiente confianza. Asi que decidi escaparme. Me fui de China cuando tenía veinte y pico años..., y después de doce años volví. Y entonces estaba más preparado. Durante todo ese tiempo en Nueva York, en los EEUU, maduré mucho. Estaba metido en el arte contemporáneo pero a la vez veía cómo funciona una sociedad democrática. Ahora veo que no tengo miedo (Ai Weiwei, CAAC, 2010).

Ai Weiwei cuando regresó a China, el año 1993, ayudó a que una nueva generación de artistas experimentales dieran a conocer su trabajo mediante una serie de tres catálogos: Black Cover Book (1994), White Cover Book (1995) y Gray Cover Book (1997). Sus propuestas -como artista, curator, crítico, arquitecto y editor-son entendidas como herramientas para espolear la sociedad en la que vive. Justamente, en relación a la función social y política del arte, Ai Weiwei explica en una entrevista con Chin-Chin Yap:

It can serve a social and political function in that it is independent and self-critical. Art is an individual expression and should encourage private thinking and self-reflection. This is the most important political attitude to have today, because governments, nations, political and economic institutions, as well as education, fashion all have powerful political goals and economic interests which normally dominate the majority of everyone's life. (...) At the same time, because you lack the means to control your lifestyle and attitude, you really become powerless in the true sense of the word (Ai Weiwei, 2003, pp. 52-53).

\section{Arte y compromiso: proyectos colaborativos}

Es preciso enmarcar los proyectos colaborativos dentro de una estética contextual, relacional, en la que la creación se vincula con prácticas colectivas, entendiendo la obra de arte como intersticio social. Aquí es preciso citar al crítico y comisario francés Nicolas Bourriaud, quien en su libro Estética relacional (Esthétique relationnelle, 1998) recoge la idea de rehabilitar la experimentación: "La posibilidad de un arte relacional (un arte que toma por horizonte teórico la esfera de las interacciones humanas y su contexto social, más que la afirmación de un espacio simbólico autónomo y privado) testimonia un giro radical de los objetivos estéticos, culturales y políticos puestos en juego por el arte moderno" (Bourriaud, 2001, p. 430). Este arte contextual, participativo, que parte principalmente de las prácticas artísticas activistas de los años sesenta y setenta del pasado siglo, da la voz al público, a la ciudadanía. Ejemplos de esta manera de entender la creación artística desde la colaboración los hallamos en varias de las propuestas artísticas de Ai Weiwei, en las que el espectador deja de ser un sujeto pasivo para devenir un sujeto activo.

En Fairytale-1001 Chairs (2007), realizada en la doceava edición de Documenta, el artista invitó a 1001 residentes chinos a que viajaran a Kassel y se pasearan por la ciudad y la muestra, en diversas tandas de 200 personas durante 8 días. Es pertinente señalar que la población de dicha ciudad oscila alrededor de unos 194.000 ciudadanos. Con esta iniciativa el artista pretendía generar una confrontación entre los habitantes de la ciudad y el grupo de visitantes chinos (seleccionados entre las 3.000 solicitudes recibidas vía internet), convirtiendo dicho trabajo en el más costoso de la historia de Documenta (el presupuesto ascendió a más de 4 millones de dólares).

En Sunflowers seeds (2010) cubrió la superficie de 1.000 metros cuadrados de la Sala de Turbinas de la Tate Modern con cinco toneladas de semillas de girasol 
realizadas artesanalmente -durante dos años y medio- por 1.600 trabajadores chinos de la región de Jingdezhen. El artista confronta de este modo las técnicas de producción ancestrales con las técnicas de producción industrial propias de la modernidad.

En Remembering (2009) dispuso nueve mil mochilas escolares en la fachada del Haus der Kunst, en Múnich, para construir la siguiente frase "Ella vivió felizmente siete años en este mundo". Dicha sentencia fue pronunciada por una de las madres cuya hija falleció en el seísmo de Sichuan (2008), denunciando así las negligencias constructivas de los edificios escolares que causaron la muerte de miles de menores durante el seísmo. Asimismo, el artista publicó en su blog la lista de nombres de los más de 5.000 niños fallecidos. Es necesario apuntar que su blog fue cerrado después de publicar tal lista y, como respuesta, Ai Weiwei recurrió a twitter para difundir la información. Tal y como expongo en el artículo "Producción colaborativa en la era digital: encuentros entre arte y política":

El artista Ai Weiwei solicitó a voluntarios, mediante las nuevas redes de comunicación, para que le enviaran su voz grabada pronunciando los nombres de los niños fallecidos, creando así una obra cooperativa. De este modo, con miles de personas leyendo en voz alta los nombres de las víctimas, el artista pretende recordar y ofrecer un singular homenaje a los fallecidos. Precisamente, la visibilidad que ofrecen las redes virtuales y el apoyo que brindó al activista Tan Zuoren, quien investigaba también los materiales empleados en los edificios escolares, provocaron que Ai Weiwei fuera golpeado por la policía, en Chengdu (en agosto de 2009), donde se hallaba para declarar a favor de Tan Zuoren. Como consecuencia de la agresión policial, dos meses antes de la inauguración de la exposición, Ai Weiwei tuvo que someterse a una operación de urgencia en Alemania a raiz de una hemorragia interna. Su prolifica actividad en la red, mediante su blog y twitter, ha sido determinante para difundir exponencialmente lo sucedido. La amplia recepción de sus denuncias, dentro y fuera de China, propició el arresto del artista el 3 de abril del 2011 bajo los cargos de una supuesta disidencia política y presuntos fraudes fiscales (Manonelles, 2013, pp. 169-170).

Ai Weiwei explica en relación a su blog:

I cannot self-censor. Because that is the only reason I have the blog. (...)I think China is at very interesting moment. Power and the centre have suddenly disappeared in the universal sense because of the Internet, global politics, and the economy. The techniques of the Internet have become a major way of liberating humans from old values and systems, something that has never been possible until today (Ai Weiwei; Obrist, 2011, p. 6).

Fairytale-1001 Chairs (2007), Remembering (2009) y Sunflowers seeds (2010) parten de la participación directa de personas sin una previa vinculación con el mundo artístico. Los residentes chinos que se convierten en turistas en Kassel, los artesanos que dan forma al proyecto Sunflower Seeds y los activistas y voluntarios que se implican en Remembering, son la columbra vertebradora de tales iniciativas. El arte y la vida se funden y en estas obras el proceso es esencial. Se busca crear nuevos espacios para compartir y reflexionar.

Con la misma voluntad de enfocar directamente diversas problemáticas sociopolíticas en Circle of Animals/Zodiac Heads (2010) evoca la historia 
transnacional, siendo dicha instalación expuesta -entre otros lugares- en La São Paulo Biennale (2010), en el Central Park de Nueva York (2011), en el Courtyard of Somerset House de Londres (2011), en la plaza de Los Angeles County Museum of Art (2011-2012), en el Taipei Fine Arts Museum de Taiwán (2012) y en el Hirshhorn Courtyard de Washington (2013). Ai Weiwei recrea las doce cabezas del zodíaco chino que realizó el jesuita Giuseppe Castiglione (1688-1766) para la fuente-reloj de los jardines del emperador Qianlong. Es importante recordar que tales esculturas fueron saqueadas y expoliadas por las tropas francesas y británicas en la Segunda Guerra del Opio en China y, posteriormente, varias de estas figuras aparecieron en una subasta de Sothesby de Hong Kong (2000) y en una subasta de Christie's en Paris (2009) desatando vivas polémicas.

También es interesante destacar, entre sus proyectos artísticos, la instalación S.A.C.R.E.D. (2013) presentada en la $55^{\mathrm{a}}$ Bienal de Venecia en la iglesia barroca Saint-Antonin. Ai Weiwei construyó los escenarios que enmarcaban sus rutinas diarias durante su estancia en prisión. En tales estancias se podían contemplar unas figuras de fibra de vidrio, que encarnaban a su persona y a los guardias que lo vigilaban (día y noche), y así el visitante podía ver a la réplica hiperrealista del artista comiendo, durmiendo y duchándose entre otras de sus actividades cotidianas. Aquí es preciso mencionar que, paralelamente, Ai Weiwei publicó en internet un videoclip en el que ridiculizaba su detención. En la misma dirección, Ai Weiwei protagonizó otro videoclip bailando el "Gangnam Style" agitando paródicamente unas esposas. Tal y como explica el periodista José Reinoso en el artículo publicado en El País "Ai Weiwei se suma al ‘estilo Gangnam’ para criticar a Pekín”:

El vídeo se llama 'Estilo Cao Ni Ma'. Este término, que literalmente significa hierba, barro, caballo, suena como 'que jodan a tu madre', y ha sido utilizado por los internautas en China y por el mismo Ai para ridiculizar el estricto control que ejerce el Gobierno sobre Internet. Pero los censores no participan del humor político de Ai Weiwei, y, pocas horas después de que fuera colgado, el vídeo fue retirado de las webs chinas. Se puede ver en Youtube, página que está bloqueada en el país asiático, aunque muchos internautas acceden a ella mediante programas informáticos que permiten saltarse el llamado 'Gran cortafuegos chino'. Cao Ni Ma fue concebido como un animal mítico, similar a una alpaca, y ha sido utilizado en otras ocasiones por Ai. El artista se ha hecho fotos desnudo, mientras salta en el aire, con un muñeco de peluche blanco que parece una alpaca cubriéndole los genitales. La colocación del animal en ese lugar estratégico evoca la frase: 'Cao Ni Ma Comité Central del Partido'(Reinoso; Ai Weiwei, 2012, 25 de octubre).

El mismo año 2013, en Hong Kong, realizó la instalación Baby Formula inspirada en la gran polémica que hubo en China cuando, en 2008, fallecieron 6 bebés y enfermaron más de 300.000 después de consumir leche en polvo contaminada con melamina. En Baby Formula dibujó un mapa de China con 1.815 latas de leche en polvo cubriendo los 89 metros cuadrados del espacio expositivo Sheung Wan Civic Centre, organizado por Para Site Art Space. El artista parte de una problemática cotidiana, puesto que muchos turistas procedentes de China continental -cuando viajan a Hong Kong y a otros países extranjeros- compran grandes cantidades de leche en polvo para bebés, hasta llegar al extremo de que en Hong Kong se ha tenido que limitar la cantidad de leche infantil que pueden llevarse los visitantes. 


\section{La recepción de la producción artística de Ai Weiwei}

\subsection{La recepción de Ai Weiwei en el marco internacional y en el contexto español}

Una vez esbozados brevemente algunos de sus trabajos más significativos hay que recalcar que tales iniciativas han suscitado y suscitan un gran interés, obteniendo una gran repercusión y convirtiéndose, actualmente, en el artista chino con mayor proyección internacional.

Si analizamos las noticias que aparecen en los periódicos españoles en relación con dicho autor, es importante remarcar que la mayoría de titulares hacen referencia directa al estereotipo de artista politizado, a su "disidencia" y su detención (el día 3 de abril de 2011) por parte de las autoridades de su país bajo la acusación de evasión de impuestos. Con todo, no se puede obviar que el arresto coincidió con su prolífera actividad en la red denunciando diversas injusticias y con una supuesta campaña de represión contra la disidencia. Tanto la prensa internacional como la española recogieron las reacciones de apoyo suscitadas por el cautiverio del artista, exigiendo su inmediata liberación los embajadores y los directores de centros museísticos entre los que destacan la Fundación Guggenheim, el Museo de Arte Moderno de Nueva York (MOMA), la Tate Modern y la Asociación de Directores de Museos de Arte. Simultáneamente, también se produjeron protestas ante las embajadas y consulados de China en Europa, Estados Unidos y Australia (Fig. 1). En relación al posicionamiento del gobierno español sobre dicho asunto es interesante recordar que el que era -en aquel momento- presidente del Gobierno, José Luis Rodríguez Zapatero, evitó pronunciarse sobre el tema en sus encuentros con los representantes oficiales de Pekín durante su estancia en China.

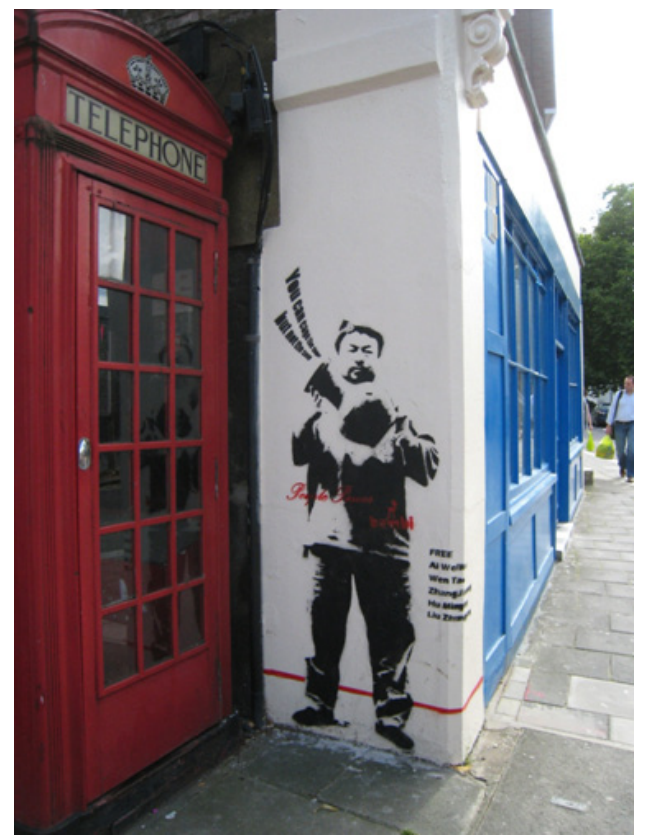

Figura 1. Graffiti en muestra de apoyo al artista. Londres, 2011. (Fotografía realizada por la autora). 
Después de 80 días de cautiverio Ai Weiwei fue liberado y entonces empezó formalmente su persecución judicial. Ha sido acusado de evasión de impuestos, defraudados a través de su estudio Beijing Fake Cultural Development, y condenado a pagar una multa de 15 millones de yuanes (1,85 millones de euros) sin poder salir de su país al haberle retirado el pasaporte. Un ejemplo de la repercusión de la detención del artista puede verse en la concepción de la obra \#aiww: El arresto de Ai Weiwei (2013), creada por Howard Brenton. \#aiww: El arresto de Ai Weiwei escenificó, en un teatro de Londres, la detención del artista, los dos meses y medio en cautividad y su liberación. La obra también pudo verse gratuitamente online el viernes 19 de abril. Howard Brenton parte del relato del propio creador, recogido por el periodista Barnaby Martin en la publicación Hanging Man, y esta iniciativa nace de la voluntad de reflexionar sobre los derechos humanos, la libertad de expresión y el papel del arte en la construcción de la sociedad global.

El encarcelamiento del artista es también uno de los reclamos de la exposición Evidence, en el museo Martin-Gropius-Bau de Berlín (de abril a julio de 2014), en la que Ai Weiwei reconstruye -a partir de su memoria- la celda en la que estuvo recluido. Tres años después de su polémica detención la muestra presenta varios trabajos del artista con los que denuncia injusticias y profundiza en experiencias vividas. La obra que recrea la celda en la que estuvo preso, de 28 metros cuadrados, se titula 81 en alusión directa a los días de cautiverio. En la exhibición también se puede visualizar un vídeo donde se ve a Ai Weiwei mientras desarrolla las actividades diarias (comer, ducharse, dormir) siempre acompañado por los guardias de seguridad.

En la entrevista que realizó José Reinoso a Ai Weiwei (Reinoso; Ai Weiwei, El País, 2011, 13 de diciembre) el artista explica que durante su cautiverio fue sometido a más de cincuenta interrogatorios esposado. En la exposición de Berlín, las esposas son transformadas en una joya de jade de grandes dimensiones. Otro de los elementos que aparecen en una de las salas de la muestra son los materiales de oficina que fueron requisados de su estudio por la policía. Es decir; cámaras fotográficas y discos duros entre otras pertenencias. También es preciso mencionar que en la exhibición Evidence se podían encontrar - para adornar una puerta- los restos de cemento y ladrillos de un taller de Ai Weiwei que fue demolido por órdenes gubernamentales.

La muestra fue inaugurada sin la presencia del artista, puesto que no posee todavía su pasaporte para poder viajar. En esta propuesta puede verse claramente la conexión entre la experiencia vital y la artística, aunque el artista no se limita a desglosar unos hechos autobiográficos, personales e íntimos, sino que en sus proyectos conecta tales vivencias con la sociedad y con la realidad de la China actual. Un ejemplo significativo de la recepción del trabajo de Ai Weiwei en China es la exclusión de sus trabajos en la exposición colectiva 15 Years Chinese Contemporary Art Award (2014), en Shanghái, debido a su posicionamiento crítico respecto el gobierno chino. Dicha exhibición aglutina más de cincuenta trabajos de los seleccionados por los premios artísticos impulsados, desde 1998, por el embajador y coleccionista suizo Uli Sigg (Ramzy, 2014, 29 de abril).

Tal y como se ha apuntado previamente Ai Weiwei suscita un gran interés mediático y los medios de comunicación inciden especialmente en el compromiso político del artista, en su activismo, recurriendo a ciertas palabras como "disidencia" 
de manera reiterativa. Si se toma como ejemplo el periódico El País es interesante prestar atención a los titulares que preceden las noticias sobre dicho artista: "Entrevista a Ai Weiwei, un artista contra la autoridad" (Reinoso, El País, 2009, 16 de mayo), "Reportaje: El 'enemigo' de China ¿Dónde está Ai Weiwei? El arresto del cotizado artista, y disidente ha hecho saltar las alarmas en la comunidad internacional, pero Pekín le acusa de delitos económicos" (Reinoso, El País, 2011, 24 de abril), "Una obra de Ai Weiwei recorre el mundo mientras el artista sigue detenido en China. El alcalde de Nueva York homenajea al disidente chino en la inauguración de las esculturas a las puertas de Central Park" (Agencias, El País, 2011, 5 de junio), "El Gobierno chino libera bajo fianza al artista y disidente Ai Weiwei" (Reinoso, El País, 2011, 22 de junio).

En otros periódicos como La Vanguardia. El Público y el $A B C$ numerosos titulares adoptan la misma dirección: "Critica Ai Weiwei su detención en un video musical" (DPA, La Vanguardia, 2013, 22 de mayo), "Encarcelamiento de Ai Weiwei visita Bienal de Venecia. En la instalación 'S.A.C.R.E.D." (Super, Accusers, Cleansing, Ritual, Entropy, Doubt) el artista chino muestra sus días en prisión, en un lugar secreto durante 81 días" (Agencia Efe, Vanguardia, 2013, 30 de mayo), "Wei wei no se calla. El artista denuncia en su cuenta de Twitter las torturas de uno de sus colaboradores encarcelados y cuestiona la autoridad de Pekín" (Brunat, D., El Público, 2011, 9 de agosto), "China reclama al artista Ai Weiwei 1,7 millones de euros en impuestos" (Agencias, El Público, 2011, 1 de noviembre), "Ai Weiwei en el país de sus pesadillas. El polémico artista chino exhibe en París su obra fotográfica" (Vicente, El Público, 2012, 22 de febrero), "Ai Weiwei: "Soy un peligro porque soy imprevisible"' (Díez, $A B C, 2013,12$ de febrero).

Yendo más allá, en China Files, se publicó el artículo "El artista mártir de Occidente" (Tobón, 2011, 24 de mayo) en el que se ahondaba en la construcción del arquetipo de "mártir":

Su imagen subversiva y rebelde, que se enfrenta solitariamente al fuerte aparato de la nueva segunda potencial mundial, lo han convertido en el mártir preferido de occidente. Ai de una u otra forma es el luchador que un país como Estados Unidos, que tanto critica a China por violación de derechos humanos, desea y busca promover (Tobón, 2011, 24 de mayo).

Esta atracción por la subversión y por la imagen de artista politizado, sea dicho estereotipo construido por el propio artista o bien "otorgado" por los medios y el circuito del arte, conlleva una amplia cobertura mediática y un activo seguimiento de su detención, su liberación y de sus producciones artísticas. Esta fascinación por el "personaje" de Ai Weiwei y sus propuestas irreverentes no se puede desvincular de la atracción por el efectismo propio de las estrategias del marketing que son utilizadas frecuentemente en los medios de comunicación y, también, en los centros museísticos. Pues hay que tener presente que la revista Art Review ubica a Ai Weiwei entre los primeros de la lista de las personas más "poderosas e influyentes" del mundo del arte del 2012. Ai Weiwei deviene un icono, un "objeto de deseo" tal y como expone la crítica de arte Barbara Pollack en su libro The wild, wild east: an American art critic's adventures in China (2010): 
Yet he is a bit of ham, performing for the crowds of reporters who come to his door and have made his face into an icon of Chinese art, everywhere from the BBC to Vanity Fair. Charismatic as he is, he is also the least likely object of desire in Beijing with his big belly flopping over his baggy pants... (Pollack, 2010, pp. 24-25).

\subsection{Resistencia y tradición en el CAAC de Sevilla}

Dentro de los parámetros previamente bosquejados es interesante poner atención a la recepción de la producción artística de Ai Weiwei en España. Puesto que es uno de los creadores contemporáneos procedentes de China que más presencia e impacto ha logrado tanto en los espacios expositivos españoles como en los medios de comunicación.

Una muestra de ello es la primera exposición individual que le organizaron en España, comisariada por Hans-Ulrich, en el espacio Ivorypress Art+Books en Madrid (2009). También hay que considerar la instalación With milk, find something everybody can use (2009), en la que inundó de café con leche las piscinas del Pavellón Mies van der Rohe de Barcelona, y la primera exposición museística en el Centro Andaluz de Arte Contemporáneo (CAAC), en el conjunto monumental de la Cartuja de Sevilla (2013), comisariada por Juan Antonio Álvarez y Luisa Espino. Además, en 2013, en España se estrenó la película documental sobre su vida y su obra Never Sorry, dirigida por Alison Klayman.

En las exposiciones en el espacio Ivorypress Art+Books (2009) y en el CAAC (2013) el artista exhibió varias de sus piezas más emblemáticas, además de aglutinar distintos lenguajes artísticos que subrayan el carácter holístico de su trayectoria. Son exposiciones panorámicas que introducen la creación de dicho artista en un marco en el que su obra no es conocida. Tales muestras son de un gran interés porque asumen la responsabilidad y la labor de hacer un trabajo introductorio aunque, en esta aproximación general, existe el riesgo de repetir y perpetrar los titulares que siempre acompañan al mediático artista. De este modo el espectador se encuentra que tanto en el discurso de las exposiciones mencionadas como en las notas de prensa y en la repercusión en los medios de comunicación se insiste principalmente en subrayar su papel de agitador político. Aquí es necesario tener presente que la mayoría de exposiciones de arte chino contemporáneo, programadas en el marco euroamericano, comparten el mismo interés de resaltar la naturaleza politizada y disidente del arte experimental chino. La rebeldía y la censura ayudan a atraer a un público que desconoce este arte, puesto que la subversión se convierte en un reclamo para cautivar a la audiencia en un contexto en el que todavía se proyectan las reminiscencias de la "Guerra fría". En dicho escenario, el arte que critica a los "viejos adversarios" resulta atractivo, se reproducen los paradigmas conocidos y, en tal contexto, cabría plantear la necesidad de generar espacios en los que poder recibir lo que aún no se ha dicho ni escuchado.

Seguidamente nos centraremos en la exposición monográfica Resistencia y tradición de Ai Weiwei, programada en el Centro Andaluz de Arte Contemporáneo. La declaración a la prensa del director del CAAC es especialmente ilustrativa: "Será la exposición más mediática" (Grosso, El Correo, 2012, 7 de febrero). La exhibición se inauguró sin la presencia del artista tal y como se expone en un artículo en $E l$ 
País "Sevilla rompe el cerco de Ai Weiwei. El CAAC es el primer museo español en dedicar una muestra al creador perseguido por Pekín":

Es la primera dedicada por un museo español al artista chino. Se inauguró por fin hoy - estuvo programada para el pasado septiembre, pero se cayó del cartel por falta de dinero - en el sevillano Centro Andaluz de Arte Contemporáneo (CAAC) y sin la presencia de Ai Weiwei. Tampoco pudo participar en el montaje, pese a que lo ha supervisado desde su estudio en Pekín; las autoridades no le permiten salir de China, donde recibe el tratamiento de "enemigo público" y se enfrenta a acusaciones por evasión fiscal y pornografia (Gallego; Grosso, 2012, 7 de febrero).

Es preciso volver a recalcar cómo la construcción del arquetipo de artista politizado es omnipresente. Disidente, creador perseguido, enemigo público, son varios de los calificativos que frecuentemente son utilizados para referirse a Ai Wewei. Queda claro que la polémica forma parte de las presentaciones que suelen acompañar el trabajo de dicho artista. Dentro de este marco -y con tales "expectativas"- cabría preguntarse ¿qué se muestra en el Centro Andaluz de Arte Contemporáneo (CAAC) de la extensa producción del artista?

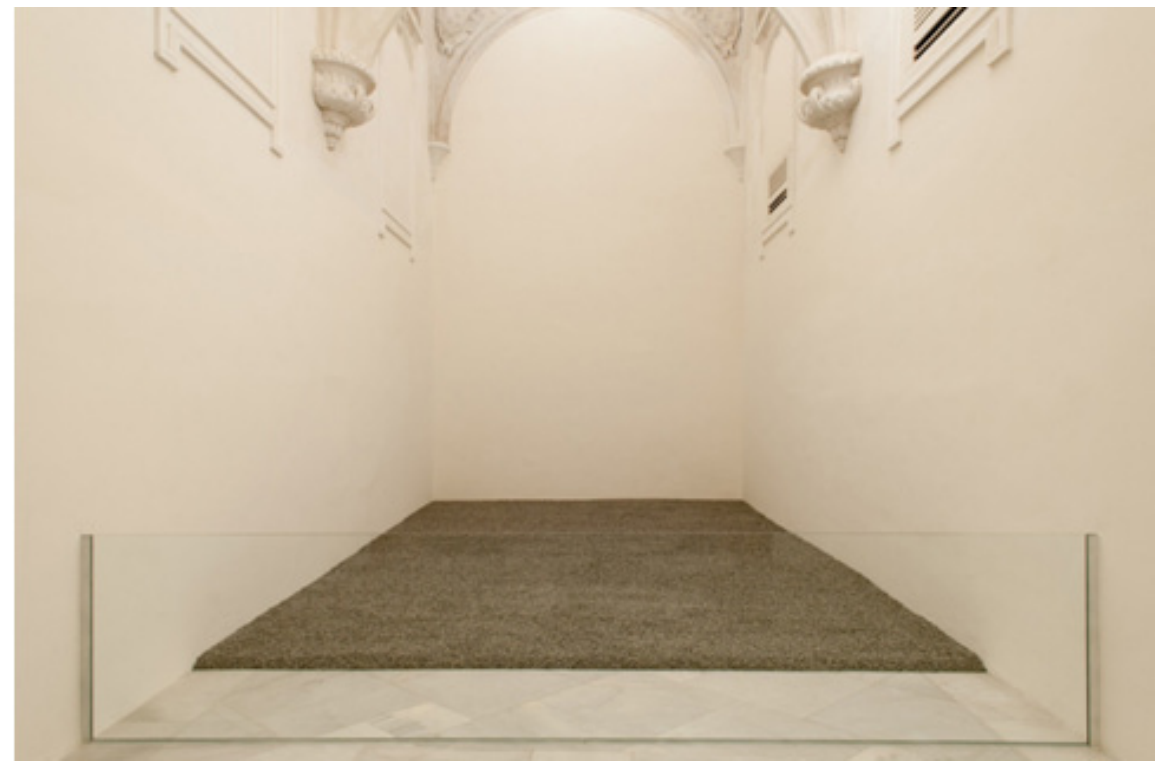

Figura 2. Ai Weiwei, Sunflower Seeds, 2009. Vista de la instalación en el CAAC. (Imagen facilitada por el CAAC).

En Sevilla se exhibieron una selección de vídeos de sus acciones y de textos de su prolífico blog (iniciado el año 2005), además de obras como Sunflower Seeds (2009) (Fig. 2), expuesta previamente en la Tate Gallery de Londres. Otra instalación que formaba parte de la muestra fue Descending Light (2007) (Fig. 3), en la que el autor alude al mítico cuadro Desnudo bajando la escalera de Duchamp y presenta una gran lámpara con cristales rojos engarzados -que nos recuerdan al color institucional de China- que parece haber caído violentamente en el suelo. 


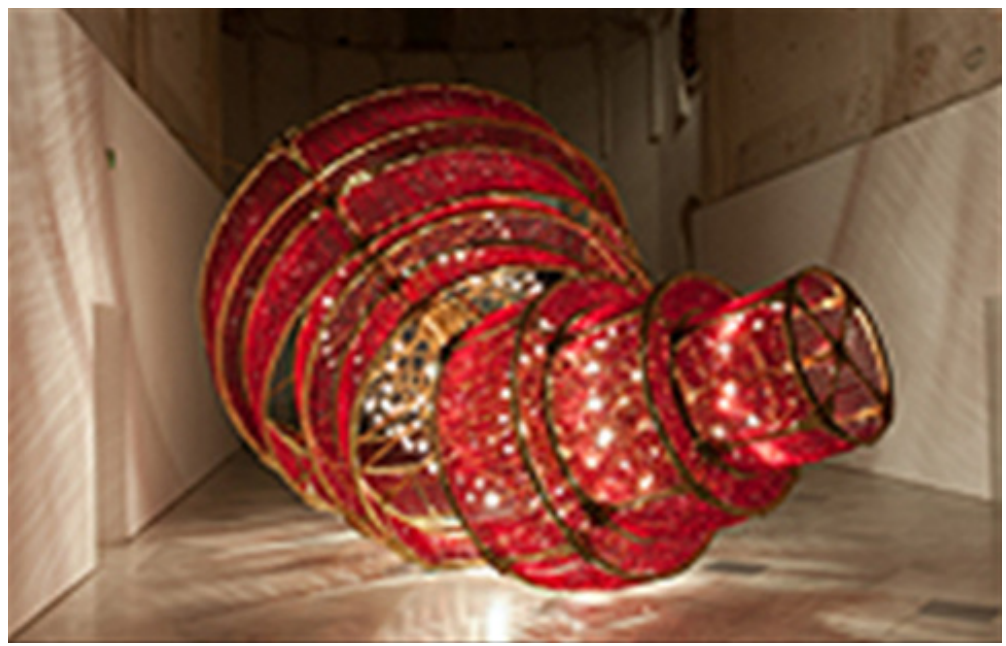

Figura 3. Ai Weiwei. Descending Light, 2007. Vista de la instalación en el CAAC. (Imagen facilitada por el CAAC).

También pudo contemplarse la instalación Ghost Gu Coming Down the Mountain (2005) (Fig. 4), realizada en colaboración con el artista de origen rumano Serge Spitzer, compuesta por 96 vasijas pintadas en blanco y rojo en las que puede verse representada una escena que evoca la leyenda de Guiguzi $(\mathrm{Gu})$.

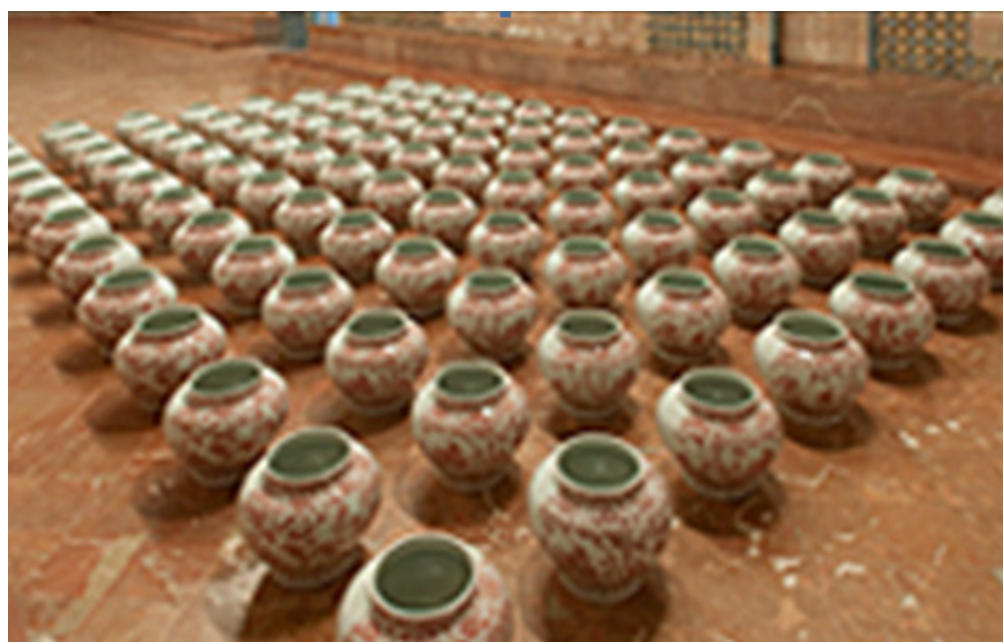

Figura 4. Ai Weiwei \& Serge Spitzer. Ghost Gu Coming Down the Mountain, 2005. Vista de la instalación en el CAAC. (Imagen facilitada por el CAAC).

Durante el transcurso de la exhibición una mujer tropezó con una de las piezas de dicha instalación rompiendo una de las urnas expuestas. La misma acción-de manera irónica- también se podía visionar en la muestra en la performance Dropping a Han Dynasty Urn (1995), en la que Ai Weiwei arrojó contra el suelo una vasija milenaria, 
cuestionando así el valor y el culto de ciertas obras y las leyes de protección del patrimonio. Hay que señalar que tal incidente despertó un nuevo foco de atención e interés de los medios de comunicación hacia la muestra.

Este incidente puede relacionarse con la decisión del artista Máximo Caminero de hacer añicos un jarrón de la dinastía Han, de 2000 años de antigüedad, que formaba parte de la instalación Colored Vases expuesta en la muestra temporal Ai Weiwei: According To What? (de diciembre de 2013 a marzo de 2014). Aunque Máximo Caminero no tropezó azarosamente con la pieza, como la señora de Sevilla, sino que se inspiró en la acción Dropping a Han Dynasty Urn (1995) de Ai Weiwei para denunciar que en el Pérez Art Museum Miami (PAMM) no se apoya ni se exhiben artistas locales. Los servicios de seguridad del Museo detuvieron al artista por los daños causados a la obra, valorada en unos 730.000 euros según los responsables de la institución, acusándolo de vandalismo. El titular de la publicación El Huffington Post recoge la noticia con las siguientes palabras: "Un artista destroza un jarrón de Ai Weiwei de 1 millón de dólares como protesta" (2014, 18 de febrero). Ai Weiwei, en respuesta a este incidente, argumentó en una entrevista "I don't encourage anyone to protest by destroying other people's property" y también añade respecto las intenciones de la destrucción de las urnas que "It's very different(...)What I destroyed didn't belong to anybody else" (Silk, 2014, 18 de febrero). Es sorprendente que aquí utilice conceptos como la "propiedad" un autor que trabaja frecuentemente con las dialécticas entre lo verdadero y lo falso, los originales y sus copias.

Regresando al discurso expositivo propuesto por el Centro Andaluz de Arte Contemporáneo, en la exposición monográfica de Ai Weiwei Resistencia y tradición, es pertinente citar el dossier de prensa en el que se lanzan las siguientes coordenadas para aproximarse a la muestra:

La gran mayoría de sus trabajos participan, por tanto, de ese tiempo político y de ese espacio cultural y, al igual que ellos, están en continua y vertiginosa trasformación. Esta es una de sus características: utilizar y pervertir la tradición cultural china y la artística occidental como actos de resistencia política, para los que se vale de todas aquellas herramientas que le son útiles, ya provengan de una u otra tradición, a las que une como singularidad la rapidez en el empleo de las redes sociales que el capitalismo informacional, por utilizar el término acuñado por Manuel Castells, ha ido proporcionando.

Esta "resistencia política" queda perfectamente esbozada y recogida tanto en los discursos articulados por los medios de comunicación como en las exhibiciones que Ai Weiwei protagoniza, ya sea en el marco español o bien en un escenario internacional.

Si partimos de los artículos generados a partir de la exposición de Ai Weiwei en el CAAC puede verse como numerosos titulares evocan las directrices mencionadas previamente. Cito a modo de ejemplo: "El arte y la disidencia de Ai Weiwei recalan en el CAAC" (Ramos, Diario de Sevilla, 2013, 9 de febrero), "Disidencias de porcelana" (Ramos, Diario de Sevilla, 2013, 1 de febrero), "Ai Weiwei, disendencia en un monasterio" (Díaz, El Mundo Sevilla, 2013, 1 de febrero),"Sevilla rompe el cerco de Ai Weiwei" (Seisdedos, El País, 2013, 1 de febrero), "Poesía y política en la obra de Ai Weiwei" (Glez-Santiago, Ideal de Granada, 2013, 1 de febrero), "El arte del disidente Ai Weiwei en el monasterio de la Cartuja" (Agencia Europa Presss, 
La Razón de Sevilla, 2013, 1 de febrero), "Ai Weiwei: "Soy un peligro porque soy imprevisible" (Díez, ABC Sevilla, 2013, 10 de febrero), "El artista como crítico y catalizador social" (Bosco, Diario de Sevilla, 2013, 4 de marzo). Si profundizamos en el artículo "El arte y la disidencia de Ai Weiwei recalan en el CAAC", de Charo Ramos, puede verse cómo la aproximación a Ai Weiwei se centra principalmente en señalar su rol de agitador. El estereotipo de artista politizado se repite y el término "disidente" y su carácter contestatario aparecen en el artículo: "Sevilla agranda ahora la reivindicación española de este genio contestatario detenido en 2011 por las autoridades chinas, que no puede salir de su país pero sigue sin morderse la lengua: recientemente criticó el Nobel de Literatura otorgado a Mo Yan, su compatriota, al que tachó de "guardián del sistema dictatorial"."

Si nos centramos en el interés mediático, en otros artículos se hace hincapié en la expectación que suscita el artista: "Ai Weiwei, el arte y el atractivo turístico" (Agencia, El Mundo, 2013, 10 de enero), "Expectación ante la primera exposición de Ai Weiwei en España" (Glez.-Santiago, Sur, 2013, 24 de enero), "Weiwei Superstar maravilla en Sevilla" (Pulido, ABC Andalucia, 2012, 1 de febrero), "Ai Weiwei compromiso superstar" (Luque; Díez, El Correo de Andalucía, 2013, 1 de febrero), "Ai Weiwei ha convocado ya en el CAAC a más de 40.000 personas" (Agencia, Diario de Sevilla, 2013, 8 de junio). En esta dirección, para subrayar el gran éxito de la propuesta expositiva, el Diario de Sevilla afirmaba: "Ha sido la exposición de arte contemporáneo producida por el CAAC más visitada" (Diario de Sevilla, 2013, 1 de julio).

La remarcable afluencia de público -con 45.000 visitantes- y el seguimiento que consigue dicho artista genera múltiples lecturas e interpretaciones, siendo una de ellas la que apunta Elena Vozmediano en "Ai Wei wei, monástico y mundano" (Vozmediano, El Mundo, 2013, 8 de ferbero): "El mercado del arte internacional creó a Ai Weiwei como estrella artística. Si no fuera mediático no cotizaría. Si no cotizara no sería mediático y no podría proyectar sus denuncias".

\section{Estrategias subversivas}

Esta transformación del artista en un "objeto de deseo", como citábamos previamente, nos conduce a la fortuna crítica de los creadores y las obras. Aquí es pertinente citar al sociólogo Pierre Bourdieu quien profundiza en el pensamiento relacional, remarcando la importancia de las interrelaciones y conexiones que se crean entre el individuo y la comunidad a la que éste pertenece. Asimismo, en su libro Creencia artística y bienes simbólicos, elementos para una sociología de la cultura (2003), recalca la importancia de la creencia para crear y dar valor a ciertas obras artísticas. Bourdieu se aproxima a la dialéctica entre los productores y los consumidores de los bienes y sus respectivas relaciones de fuerza, tal y como sintetiza Alicia B. Gutiérrez en la introducción:

(...) dentro de una lógica de mercado, es importante la existencia de intermediarios, algunos de los cuales actúan como instancias de consagración y legitimación especificas del campo, y el surgimiento de la diversificación y de la competencia entre productores y consumidores (Bourdieu; Gutiérrez, 2003, p.11). 
Queda claro que de manera implícita o explícita Ai Weiwei deviene un modelo de artista político, al amalgamar la experiencia artística con la vital y al entender la vida como compromiso.

Llegados a este punto es oportuno recordar cómo el reconocido curador y comisario Harald Szeemann remarcó que lo que más le había interesado del arte chino experimental era su espíritu reivindicativo, pues en Occidente desde finales de los sesenta -según su opinión- no había habido un arte subversivo. Tal afirmación suscitó críticas de curadores como Zhu Qi, quien lamenta que la fascinación hacia lo "político" limita la aproximación a la gran pluralidad de propuestas artísticas restringiéndola a una mirada preconcebida (Manonelles, 2011, p. 18-19). Estas afirmaciones las lanzaban Harald Szeemann y Zhu Qi a inicios del nuevo milenio y más de una década después -si hablamos de la recepción del arte contemporáneo chino- puede verse que el interés por lo subversivo continúa plenamente vigente.

Mary Bittner Wiseman, en su artículo "Subversive Strategies in Chinese AvantGarde Art" (2007), continua ahondando en este tema y recalca que el interés del público occidental hacia lo disidente, hacia la "otredad", responde a unos parámetros predefinidos. La autora escoge como ejemplo la polémica que suscitó en Inglaterra la obra Link of the body (2000), de Peng Yu y Sun Yuan, en la que la pareja de artistas realiza una transfusión de sangre a los fetos de unos siameses fallecidos. Mary Bittner Wiseman contrapone la percepción de la audiencia inglesa de la acción -considerada como una ofensa al buen gusto y a la decencia- con el premio que dicha obra recibió de la plataforma Contemporary Chinese Art Awards en 2002. Es preciso mencionar que Ai Weiwei fue el editor de la publicación Chinese artists, texts and interviews, Contemporary Chinese Art Awards 1998- 2002 (2002), y entre los entrevistados están precisamente Peng Yu y Sun Yuan. Tales premios fueron creados en 1997 por el coleccionista suizo Uli Sigg, siendo Ai Weiwei uno de los miembros del jurado.

Queda claro que no todo lo "subversivo" es bienvenido en el marco euroamericano. Como ya se ha apuntado, en una escena todavía impregnada por las dinámicas de la Guerra fría, la insurrección que resulta atractiva es la que critica, cuestiona o se mofa del poder monolítico - en el caso que nos ocupa- de China. Cito las palabras de la autora:

This leads to the thought that the avant-garde in the twenty-first century is speaking to something distinctively Chinese and that global audiences welcome this art for its difference, its Chinese-ness, but only so long as the expressed attitudes toward the subject matter do not offend their own local codes of what is acceptable (Bittner Wiseman, 2007, p. 110).

\section{Conclusiones}

El registro de la crítica política de Ai Weiwei encaja perfectamente dentro de los códigos del mundo "occidental". Otra cuestión que cabría preguntarse es si el éxito de Ai Weiwei desactiva el efecto combativo de sus obras puesto que, tal y como se ha señalado previamente, existe una estrecha relación entre la dimensión mediática del creador, su alta cotización y la visibilidad alcanzada desde la cual puede articular denuncias. 
Bosquejado este recorrido es necesario, para concluir, volver a las palabras del propio artista cuando en una entrevista responde lo siguiente a la pregunta de si el arte debe ser un arma política:

El arte me ayuda a expresar mis opiniones politicas, pero mis opiniones políticas me ayudan a estructurar mi arte. El arte no sólo debe definir nuevas propuestas estéticas, morales y filosóficas, sino también las políticas para alcanzarlas. Durante mi arresto los policías me preguntaron: ¿por qué no te dedicas a tu arte y dejas de criticar al gobierno? Pero, ¿cómo es posible que hay a valores humanos, como el arte o la libertad de expresión, sin política (Díez; Ai Weiwei, 2013, 10 de febrero).

La polémica y la ambivalencia (propia de un tándem en el que se combina el éxito con el activismo), lejos de disolverse, quedan servidas. Con todo, más allá de las controversias es importante insistir que -en el marco esbozado- el arte deviene un barómetro de la sociedad. Es decir, el arte se transforma en una herramienta para llamar la atención, para enfocar determinadas problemáticas y dar que pensar.

\section{Referencias}

Ai Weiwei (2011). Ai Weiwei speaks with Hans Ulrich Obrist. London: A penguin special.

Ai Weiwei (2003). Works: Beijing 1993-2003. China: Timezone8.

Bittner Wiseman, M. (2007). Subversive Strategies in Chinese Avant-Garde Art. The Journal of Aesthetics and Art Criticism, 65, (1), 109-119.

Bourriaud, Nicolas (2001): Estética relacional. En Blanco, P., Carrillo, J., Claramonte, J. y Expósito, M. (Eds.), Modos de hacer, arte crítico, esfera pública y acción directa (pp. 427-446). Salamanca: Universidad de Salamanca.

Bourdieu, P. (2003). Creencia artística y bienes simbólicos, elementos para una sociología de la cultura. Buenos Aires: Aurelia Rivera grupo editorial.

Díez, P., Ai Weiwei, (2013, 10 de febrero). Ai Weiwei: "soy un peligro porque soy imprevisible". ABC Sevilla. Recuperado de http://www.abc.es/cultura/ arte/20130210/abci-weiwei-201302102252.html

Gallego, J., Grosso, N. (2012, 7 de febrero). Una visitante rompe una valiosa vasija de Ai Weiwei en el CAAC. El Correo. Recuperado de http://elcorreoweb. es/2013/04/12/una-visitante-rompe-una-valiosa-vasija-de-ai-weiwei-en-el-caac/

Manonelles, L. (2013). Producción colaborativa en la era digital: encuentros entre arte y política. En Crespo, J. L. (Ed), Estudios sobre Arte Actual (163176). Málaga: Universidad de Málaga. Recuperado de http://dialnet.unirioja. es/servlet/articulo;jsessionid=2D8C219C60368AF74B7335FFBE2B772D. dialnet 02 ? codigo $=4347033$

Manonelles, L. (2011). Arte experimental en China, conversaciones con artistas. Barcelona: Bellaterra.

Pollack, B. (2010). The wild, wild east: an American art critic's adventures in China. China: Timezone8. 
Ramos, C. (2013, 9 de febrero). El arte y la disidencia de Ai Weiwei recalan en el CAAC. Diario de Sevilla. Recuperado de http://www.europasur.es/article/ ocio/1433976/arte/y/la/disidencia/ai/weiwei/recalan/caac.html

Ramzy, A. (2014, 29 de abril). Ai Weiwei Erased From Show in Shanghai. The New York Times. Recuperado de http://artsbeat.blogs.nytimes.com/2014/04/29/aiweiwei-erased-from-show-in-shanghai/? $\mathrm{php}=$ true \& type $=$ blogs \& $\mathrm{r}=0$

Reinoso, J., Ai Weiwei. (2009, 16 de mayo). Ai Weiwei, un artista contra la autoridad. El País. Recuperado de http://elpais.com/diario/2009/05/16/ babelia/1242428769 850215.html

Reinoso, J., Ai Weiwei. (2011, 13 de diciembre). En China no hay ley. El País. Recuperado de http://internacional.elpais.com/internacional/2011/12/13/ actualidad/1323793258 119982.html

Reinoso, J., Ai Weiwei. (2012, 25 de octubre). Video de Ai Weiwei bailando el "Gangnam Style". El País. Recuperado de http://videos.starmedia.com/noticias/ video-ai-weiwei-bailando-gangnam-style.html

Seisdedos, I. (2013, 31 de enero). Sevilla rompe el cerco de Ai Weiwei. El País. Recuperado de http://cultura.elpais.com/cultura/2013/01/31/ actualidad/1359630813 579022.html

Silk, R. (2014, 18 de febrero). Ai Weiwei Sees No Irony After Vase Smashed. China Real Time blog, WSJ. Recuperado de http://blogs.wsj.com/chinarealtime/2014/02/18/ ai-weiwei-sees-no-irony-after

Tobón, N. (2011, 24 de mayo). El artista mártir de occidente. China files. Recuperado de http://china-files.com/es/link/10272/el-artista-martir-de-occidente

Entrevista en diciembre de 2010 a Ai Weiwei. (2013, 31 de enero). Dossier prensa CAAC (Centro Andaluz de Arte Contemporáneo). Recuperado de http://www. juntadeandalucia.es/cultura/caac/programa/aiweiwei13/frame.htm\#pre

2013 Power 100. A ranked list of the contemporary artworld's most powerful figures (2014, 15 de junio). Art Review. Recuperado de http://www.artreview100. com/2012-power-100/

Una visitante rompe una de las vasijas de Ai Weiwei expuestas en Sevilla (2013, 11 de abril). El Pais. Recuperado de http://ccaa.elpais.com/ccaa/2013/04/12/ andalucia/1365762020 105163.html

Un artista destroza un jarrón de Ai Weiwei de 1 millón de dólares como protesta (2014, 18 de febrero). EL Huffington Post. Recuperado de http://www.huffingtonpost. es/2014/02/18/ai-weiwei-vasija-xyz1_n_4807483.html?utm_hp_ref=spain 